\title{
Sea Surface Flow Estimation via Ensemble-based Variational Data Assimilation
}

\author{
Shengze Cai ${ }^{1}$, Etienne Mémin ${ }^{2}$, Yin Yang ${ }^{3}$, Chao $\mathrm{Xu}^{1}$ \\ ${ }^{1}$ State Key Laboratory of Industrial Control Technology and the Institute of Cyber-Systems \& Control, Zhejiang \\ University, Hangzhou, 310027 Zhejiang, China. \\ ${ }^{2}$ National Institute for Research in Computer Science and Control (INRIA), Campus Universitaire de Beaulieu, 35042 \\ Rennes, France. \\ ${ }^{3}$ National Research Institute of Science and Technology for Environment and Agriculture (IRSTEA), 17 Avenue de \\ Cucillé, 35000 Rennes, France.
}

\begin{abstract}
In this paper, we propose a data assimilation method for consistently estimating the velocity field$\mathrm{s}$ from a whole image sequence depicting the evolution of sea surface temperature transported by oceanic surface flow. The estimator is conducted through an ensemble-based variational data assimilation, which is designed by combining the advantages of two approaches: the ensemble Kalman filter and the variational data assimilation. This idea allows us to obtain the optimal initial condition as well as the full system trajectory. In order to extract the velocity fields from fluid images, a surface quasi-geostrophic model representing the generic evolution of the temperature field of the flow, and the optical flow constraint equation derived from the image intensity constancy assumption, are involved in the assimilation context. Numerical experimental evaluation is presented on a synthetic fluid image sequence.
\end{abstract}

\section{Introduction}

Motion estimation techniques have become increasingly significant in the study of fluid dynamics. In particular, extracting the dense velocity fields from image sequences, which allows the researchers to get a deeper insight into the complex fluid flows, plays a important role in numerous application domains, ranging from experimental fluid dynamics to geophysical flow analysis in environmental sciences.

In recent years, the research on fluid motion estimation has received a great deal of attention from the computer vision community. One of the promising directions of this topic is to apply the variational optical flow method. First proposed by Horn and Schunck [1], optical flow has been intensively studied $[2,3,4]$. In general, these methods are conducted by minimizing an objective functional composed of the brightness constancy assumption (data term) and a spatial coherency assumption (regularization term). Fluid-dedicated estimators based on the optical flow framework have been elaborated in several studies [5, 6, 7, 8]. For instance, a data term based on the integrated continuity equation and a second-order div-curl regularizer has been proposed in [5], for preserving the divergence and the vorticity of the flow. Recently, the authors of [8] suggest to replace the optical 
flow constraint with a structural sub-grid transport equation, which takes into account the small-scale velocity component of the turbulent flow. These estimators provide decent results on fluid motion analysis. However, they mostly provide the average velocity field between two successive images, with no guaranty to recover a consistent physical motion trajectory over the whole sequence.

To obtain a global spatiotemporal motion field, the optimal control strategy or stochastic filtering framework has been considered [9, 10, 11, 12]. A constrained minimization problem which is conducted for rigid motion estimation is proposed in [9]. The objective functional in [9], subject to the optical flow constraint (OFC) equation, consists of a simple observation model and a spatiotemporal smoothness constraints on the velocity field. Another work [11] suggests a similar optimal control scheme where the velocity field is subject to a stochastic representation of OFC equation and a different smoothness constraint. However, complete numerical tests remain to be done for that model. As for fluidic images, a variational assimilation method expressed as the minimization of a global spatiotemporal functional was proposed in [10]. In this context, [10] introduces a representation of Navier-Stokes equation instead of relying on additional smoothing functions, which provide physical meaning of the estimated motion fields. Note that the optimization processes in these works are realized through the introduction of adjoint techniques [13].

In this paper, we propose a framework for the recovering of a dynamically consistent flow velocity at the ocean surface via an ensemble-based variational data assimilation (referred to as EnVar hereafter). This method, based on [14] and [15], introduces an empirical ensemble-based background error covariance in the objective functional. A great advantage of doing so, compared to variational methods [10], is that the tangent linear and adjoint models can be avoided in the minimization process. On the other hand, compared to ensemble Kalman filter (EnKF) methods, this algorithm directly provides a smoothing better suited for flow estimation purpose. This framework, introduced in Section 2, allows to estimate a consistent motion field based on an initial background guess and the measured image sequence. We will rely in this work on a simplified oceanic dynamical model, namely the surface quasi-geostrophic (SQG) model [16, 17], representing the evolution of a temperature scalar field at the oceanic surface. This dynamics will be considered as a strong dynamical constraint in the variational assimilation problem. The OFC equation, which describes the evolution in terms of the observed luminance function, is used as the observation model. The details of these implementation$\mathrm{s}$ are presented in Section 3. An experimental evaluation is carried out in this study (Section 4) to validate the performance of the proposed estimator.

\section{Ensemble-based Variational Data Assimilation}

The enhanced ensemble-based variational data assimilation is composed of several procedures [15]: a) generation of the ensemble initial condition and observation; b) minimization of the preconditioned objective functional based on the approximated background error covariance matrix; c) update of the initial condition and the corresponding ensemble perturbation matrix.

\subsection{Standard incremental variational data assimilation}

Here we first introduce in general terms the definitions of the state equations. Let $\boldsymbol{X}(\boldsymbol{x}, t), \boldsymbol{Y}(\boldsymbol{x}, t)$ be the state vector and the observation vector, respectively, both defined over the physical spatial domain $\Omega$ and the time range $\left[t_{0}, t_{f}\right]$. The overall dynamical system can be represented by the following 
equations:

$$
\left\{\begin{array}{l}
\partial_{t} \boldsymbol{X}(\boldsymbol{x}, t)+\mathbb{M}(\boldsymbol{X}(\boldsymbol{x}, t))=v(\boldsymbol{x}, t), \\
\boldsymbol{X}\left(\boldsymbol{x}, t_{0}\right)=\boldsymbol{X}_{0}(\boldsymbol{x})+\eta(\boldsymbol{x}) \\
\boldsymbol{Y}(\boldsymbol{x}, t)=\mathbb{H}(\boldsymbol{X}(\boldsymbol{x}, t))+\boldsymbol{\varepsilon}(\boldsymbol{x}, t)
\end{array}\right.
$$

The first and second equations describe the evolution of the state variables through the operator $\mathbb{M}$, as well as the initial background condition $\boldsymbol{X}_{0}(\boldsymbol{x})$. The last equation, called observation or output model, links the measurements $\boldsymbol{Y}(\boldsymbol{x}, t)$ to the state vector $\boldsymbol{X}(\boldsymbol{x}, t)$ by the operator $\mathbb{H}$. Let $\varphi_{t}\left(\boldsymbol{X}_{0}\right)$ denote the trajectory of state depending on the initial condition $\boldsymbol{X}_{0}$, then we have

$$
\varphi_{t}\left(\boldsymbol{X}_{0}\right)=\boldsymbol{X}_{t}=\boldsymbol{X}_{0}+\int_{t_{0}}^{t} \mathbb{M}(\boldsymbol{X}) \mathrm{d} s
$$

The control functions $v, \eta$ and $\varepsilon$ in (1) which represent error terms of the equations are associated to the covariance matrices $Q, B$ and $R$, respectively. In this work we will adopt a so-called strong constraint assimilation strategy, which relies on a perfect dynamical model assumption with no noise $(v(x, t)=0)$. Hereafter, we assume that any two points in the spatio-temporal domain are uncorrelated. Therefore, the matrices $B(\boldsymbol{x})$ and $R(\boldsymbol{x}, t)$ are all in diagonal form. Based on the system equations, a standard variational data assimilation problem is formulated as the minimization of the following objective functional:

$$
\begin{aligned}
J\left(\boldsymbol{X}_{0}\right)= & \frac{1}{2}\left\|\boldsymbol{X}\left(\boldsymbol{x}, t_{0}\right)-\boldsymbol{X}_{0}(\boldsymbol{x})\right\|_{B}^{2} \\
& +\frac{1}{2} \int_{t_{0}}^{t_{f}}\|\mathbb{H}(\boldsymbol{X}(\boldsymbol{x}, t))-\boldsymbol{Y}(\boldsymbol{x}, t)\|_{R}^{2} \mathrm{~d} t
\end{aligned}
$$

where $\|\cdot\|^{2}$ represents the $L_{2}$-norm $\|f\|_{A}^{2}=\int_{\Omega} f(\boldsymbol{x})^{T} A^{-1} f(\boldsymbol{x}) \mathrm{d} \boldsymbol{x}$. The associated optimal control problem seeks the initial condition $\boldsymbol{X}_{0}$ that yields the lowest error between the state variable trajectory and the measurements.

When the operators involved in the system are nonlinear, the assimilation procedure is usually improved by introducing a linearization of the dynamics around the current trajectory. Therefore, the optimization can be operated with respect to an incremental solution instead of the initial condition. Firstly, considering the increment between the state vector and the initial state, $\delta \boldsymbol{X}=\boldsymbol{X}-\boldsymbol{X}_{0}$, the operators $\mathbb{M}$ and $\mathbb{H}$ can be linearized around $\boldsymbol{X}_{0}$ :

$$
\left\{\begin{array}{c}
\mathbb{M}(\boldsymbol{X})=\mathbb{M}\left(\varphi_{t}\left(\boldsymbol{X}_{0}\right)\right)+\partial_{\boldsymbol{X}} \mathbb{M} \delta \boldsymbol{X} \\
\mathbb{H}(\boldsymbol{X})=\mathbb{H}\left(\varphi_{t}\left(\boldsymbol{X}_{0}\right)\right)+\partial_{\boldsymbol{X}} \mathbb{H} \boldsymbol{\delta} \boldsymbol{X}
\end{array}\right.
$$

where $\partial_{\boldsymbol{X}} \mathbb{M}$ and $\partial_{\boldsymbol{X}} \mathbb{H}$ denote the tangent linear operators of the model and observation operators, respectively. Accordingly, the dynamics in terms of the increment $\delta \boldsymbol{X}$ reads:

$$
\begin{aligned}
& \partial_{t} \delta \boldsymbol{X}(\boldsymbol{x}, t)+\partial_{\boldsymbol{X}} \mathbb{M} \boldsymbol{\delta} \boldsymbol{X}(\boldsymbol{x}, t)=0 \\
& \boldsymbol{D}(\boldsymbol{x}, t)=\partial_{\boldsymbol{X}} \mathbb{H} \boldsymbol{\delta} \boldsymbol{X}(\boldsymbol{x}, t)+\varepsilon(\boldsymbol{x}, t)
\end{aligned}
$$

where $\boldsymbol{D}(\boldsymbol{x}, t)$ is the innovation vector:

$$
\boldsymbol{D}(\boldsymbol{x}, t)=\boldsymbol{Y}(\boldsymbol{x}, t)-\mathbb{H}\left(\varphi_{t}\left(\boldsymbol{X}_{0}\right)\right) .
$$


Eventually, the cost functional with $\delta \boldsymbol{X}_{0}$ is defined as:

$$
\begin{aligned}
J\left(\delta \boldsymbol{X}_{0}\right)= & \frac{1}{2}\left\|\delta \boldsymbol{X}_{0}\right\|_{B}^{2} \\
& +\frac{1}{2} \int_{t_{0}}^{t_{f}}\left\|\partial_{\boldsymbol{X}} \mathbb{H} \delta \boldsymbol{X}(\boldsymbol{x}, t)-\boldsymbol{D}(\boldsymbol{x}, t)\right\|_{R}^{2} \mathrm{~d} t
\end{aligned}
$$

where $\delta X(x, t)=\partial_{X} \varphi_{t}\left(X_{0}\right) \delta X_{0}$. The associated minimization problem is subject to the dynamical model (5). A numerical solution to this problem can be obtained by using the adjoint method [13], which consists in the backward integral computation of an adjoint variable $\lambda(\boldsymbol{x}, t)$ :

$$
\left\{\begin{array}{l}
\lambda\left(t_{f}\right)=0 \\
-\partial_{t} \lambda+\left(\partial_{\boldsymbol{X}} \mathbb{M}\right)^{*} \lambda=\left(\partial_{\boldsymbol{X}} \mathbb{H}\right)^{*} R^{-1}\left(\partial_{\boldsymbol{X}} \mathbb{H} \delta \boldsymbol{X}(\boldsymbol{x}, t)-\boldsymbol{D}(\boldsymbol{x}, t)\right) .
\end{array}\right.
$$

The adjoint operators $\left(\partial_{\boldsymbol{X}} \mathbb{M}\right)^{*}$ and $\left(\partial_{\boldsymbol{X}} \mathbb{H}\right)^{*}$ are involved in this formalism. Then the gradient of the cost functional with respect to $\delta \boldsymbol{X}_{0}$ is readily given by

$$
\partial_{\delta X} J\left(\delta \boldsymbol{X}_{0}\right)=-\lambda\left(t_{0}\right)+B^{-1} \delta \boldsymbol{X}_{0}
$$

An iterative optimization strategy, e.g., quasi-Newton method, can be applied to compute the minimizer of (8).

\subsection{Preconditioning of the variational data assimilation}

For the standard incremental variational data assimilation, it is proved that the larger the condition number of the Hessian matrix of the cost function, the more sensitive the system with respect to errors in the estimate and the slower the minimization convergence rate. In order to avoid these problems, a preconditioned incremental form is usually adopted in most variational data assimilation systems, by introducing a change of variable from the state variable to the control variable. This process, also called control variable transformation (CVT), usually employs the square root of $B$ as the preconditioner:

$$
\delta X=B^{\frac{1}{2}} \delta Z
$$

Accordingly, by substituting (11) into (8), a modified objective functional is obtained:

$$
\begin{aligned}
J\left(\delta Z_{0}\right)= & \frac{1}{2}\left\|\delta Z_{0}\right\|^{2} \\
& +\frac{1}{2} \int_{t_{0}}^{t_{f}}\left\|\partial_{X} \mathbb{H} \partial_{X} \varphi_{t}\left(X_{0}\right) B^{\frac{1}{2}} \delta Z_{0}-D(x, t)\right\|_{R}^{2} \mathrm{~d} t .
\end{aligned}
$$

Compared with (8), this modified version removes the background error covariance $B^{-1}$ from the first term. Despite a better conditioning, minimization algorithm with the adjoint operators is still required for solving the optimization problem. Note that in order to compute the minimizer of (12), the background error covariance matrix is required. However, the actual value of $B$ is usually unknown in practice. In this paper we apply a low rank flow-dependent approximation of this matrix through an ensemble of realizations. 


\subsection{Ensemble-based variational data assimilation}

As mentioned above, the low rank approximation of the background covariance matrix is directly inspired from the ensemble Kalman filter (EnKF). The concept of ensemble refers to a set of samples of the system, in the same spirit as in Monte Carlo methods in which the evolution of the model statistics is represented by the propagation of ensemble states. Firstly, to generate the initial ensemble, a perturbation vector $\zeta$ is added to the background state:

$$
\boldsymbol{X}_{0}^{(i)}=\boldsymbol{X}_{0}+\zeta_{0}^{(i)}, \quad i=1, \ldots, N
$$

where $N$ is the number of ensemble element. Each member of the ensemble can be integrated through the dynamical model:

$$
\boldsymbol{X}_{t}^{(i)}=\varphi_{t}\left(\boldsymbol{X}_{0}^{(i)}\right), \quad i=1, \ldots, N .
$$

Therefore, now we have $N$ additional state trajectories. The main idea of ensemble-based method is to use the mean value of the samples to represent the actual unknown state. Let us define the operator $\langle f(t)\rangle=N^{-1} \sum_{1}^{N} f^{(i)}(t)$ as the ensemble mean of a quantity $f(t)$ through $N$ samples. By approximating $\left\langle\boldsymbol{X}_{0}\right\rangle$ as the real state vector, we can compute the empirical background error covariance matrix as follow:

$$
B \approx \frac{1}{N-1} \sum_{i=1}^{N}\left(\boldsymbol{X}_{0}^{(i)}-\left\langle\boldsymbol{X}_{0}\right\rangle\right)\left(\boldsymbol{X}_{0}^{(i)}-\left\langle\boldsymbol{X}_{0}\right\rangle\right)^{T}
$$

In the cost functional (12), only the square root of the background error covariance matrix $B^{\frac{1}{2}}$ is required. Let us define $B=\boldsymbol{A}_{b}^{\prime} \boldsymbol{A}_{b}^{\prime} T$, where $\boldsymbol{A}_{b}^{\prime}$ is called ensemble anomaly matrix. Then we have

$$
B^{\frac{1}{2}}=\boldsymbol{A}_{b}^{\prime} \approx \frac{1}{\sqrt{N-1}}\left(\boldsymbol{X}_{0}^{(1)}-\left\langle\boldsymbol{X}_{0}\right\rangle, \ldots, \boldsymbol{X}_{0}^{(N)}-\left\langle\boldsymbol{X}_{0}\right\rangle\right)
$$

with $\boldsymbol{A}_{b}^{\prime} \in \mathbb{R}^{n \times N}$ where the ensemble number $N$ is much smaller than the state space dimension $n$. Substituting this approximation into (12) we get

$$
\begin{aligned}
J\left(\delta Z_{0}\right)= & \frac{1}{2}\left\|\delta Z_{0}\right\|^{2} \\
& +\frac{1}{2} \int_{t_{0}}^{t_{f}}\left\|\partial_{X} \mathbb{H} \partial_{X} \varphi_{t}\left(X_{0}\right) A_{b}^{\prime} \delta Z_{0}-D(x, t)\right\|_{R}^{2} \mathrm{~d} t .
\end{aligned}
$$

The term $\partial_{X} \varphi_{t}\left(X_{0}\right) A_{b}^{\prime}$ can be regarded as the propagation of the ensemble perturbation matrix through the tangent linear dynamical model. To further formulate the ensemble-based variational data assimilation scheme, we also need to introduce the perturbation in observation space. The propagation error is transformed to observation model via

$$
\begin{aligned}
\partial_{\boldsymbol{X}} \mathbb{H} \partial_{\boldsymbol{X}} \varphi_{t}\left(\boldsymbol{X}_{0}\right) \boldsymbol{A}_{b}^{\prime} \approx & \frac{1}{\sqrt{N-1}}\left(\mathbb{H}\left(\varphi_{t}\left(\boldsymbol{X}_{0}^{(1)}\right)\right)-\mathbb{H}\left(\varphi_{t}\left(\left\langle\boldsymbol{X}_{0}\right\rangle\right)\right)\right. \\
& \left.\ldots, \mathbb{H}\left(\varphi_{t}\left(\boldsymbol{X}_{0}^{(N)}\right)\right)-\mathbb{H}\left(\varphi_{t}\left(\left\langle\boldsymbol{X}_{0}\right\rangle\right)\right)\right)
\end{aligned}
$$


Note that in this formulation the non-linearity of $\mathbb{H}$ and $\mathbb{M}$ is retained. The gradient of (17) with respect to the control variable reads:

$$
\begin{aligned}
& \partial_{\delta Z_{0}} J=\delta Z_{0}+\int_{t_{0}}^{t_{f}} \underbrace{\left(\partial_{\boldsymbol{X}} \mathbb{H} \partial_{\boldsymbol{X}} \varphi_{t}\left(\boldsymbol{X}_{0}\right) \boldsymbol{A}_{b}^{\prime}\right)^{T}} \\
& R^{-1}(\underbrace{\partial_{\boldsymbol{X}} \mathbb{H} \partial_{\boldsymbol{X}} \varphi_{t}\left(\boldsymbol{X}_{0}\right) \boldsymbol{A}_{b}^{\prime}} \delta Z_{0}-\boldsymbol{D}(\boldsymbol{x}, t)) \mathrm{d} t .
\end{aligned}
$$

Compared to the standard preconditioned incremental algorithm, the term with under brace has an empirical expression given by (18) and can be calculated outside the minimization iteration. This avoids the employment of the adjoint operator for computing the gradient of the cost functional at each iteration. Furthermore, the degrees of freedom of the control variable are considerably lowered due to the control variable transform (11). Thus the computational complexity is significantly reduced. By implementing the gradient-descent method, the minimizer $\widehat{\delta Z}_{0}$ can be estimated. Then the analysis state is given by:

$$
\boldsymbol{X}_{a}=\boldsymbol{X}_{0}+\boldsymbol{A}_{b}^{\prime} \widehat{\delta Z}_{0}
$$

In the situation where the initial background state is poorly known, the minimization process with multiple outer loops should be applied. That means the analysis state $\boldsymbol{X}_{a}$ is used as the background state for the next outer loop iteration. In this case, the ensemble perturbation matrix $\boldsymbol{A}_{b}^{\prime}$ corresponding to the new initial state should be updated as well. One option of updating the error covariance can be derived from the ensemble of analysis based on perturbed observations as in EnKF [18]. Similar to the ensemble generation of the background state (13), we generate the ensemble of observations by adding a perturbation vector:

$$
\boldsymbol{Y}^{(i)}(t)=\boldsymbol{Y}(t)+\xi^{(i)}(t), \quad i=1, \ldots, N
$$

where $\xi$ is assumed to be a normal distribution. Note that now we have the ensemble initial state and the ensemble observation with $N$ members respectively. The innovation vector of the $i$ th member is defined as

$$
\boldsymbol{D}^{(i)}(\boldsymbol{x}, t)=\boldsymbol{Y}^{(i)}(\boldsymbol{x}, t)-\mathbb{H}\left(\varphi_{t}\left(\boldsymbol{X}_{0}^{(i)}\right)\right), \quad i=1, \ldots, N .
$$

The CVT is also defined for every member of the ensemble initial state:

$$
\delta \boldsymbol{X}_{0}^{(i)}=\boldsymbol{A}_{b}^{\prime} \delta Z_{0}^{(i)}, \quad i=1, \ldots, N .
$$

A parallel realizations of minimization can be obtained:

$$
\begin{aligned}
J\left(\delta Z_{0}^{(i)}\right) & =\frac{1}{2}\left\|\delta Z_{0}^{(i)}\right\|^{2} \\
& +\frac{1}{2} \int_{t_{0}}^{t_{f}}\|\underbrace{\partial_{X} \mathbb{H} \partial_{X} \varphi_{t}\left(X_{0}\right) A_{b}^{\prime}} \delta Z_{0}^{(i)}-D^{(i)}(x, t)\|_{R}^{2} \mathrm{~d} t
\end{aligned}
$$

which provides minimizer $\widehat{\delta Z}_{0}^{(i)}$ for each ensemble member. Once the control variable at the $k$ th outer loop iteration is estimated, the updated initial ensemble field for the $(k+1)$ th iteration and its perturbation matrix read:

$$
\boldsymbol{X}_{0}^{(i), k+1}=\boldsymbol{X}_{0}^{(i), k}+\boldsymbol{A}_{b}^{\prime} \widehat{\delta Z}_{0}^{(i), k}
$$




Algorithm 1: Ensemble-based variational data assimilation algorithm

\section{Analysis Loop in a temporal window}

Initialize the background condition $\boldsymbol{X}_{0}$ and the corresponding ensemble $\boldsymbol{X}_{0}^{(i)}$.

Set convergence threshold $\tau_{o}$ and $\tau_{i}$ for outer loop and inner loop respectively.

Generate ensemble observation (21).

Define $\mathscr{X}_{0}^{(k=1)}=\left[\boldsymbol{X}_{0}^{(1)}, \ldots, \boldsymbol{X}_{0}^{(N)}, \boldsymbol{X}_{0}\right]$.

\section{Outer loop:}

for $k=1: k_{\max }$ do

Compute the state trajectory $\mathscr{X}^{k}(t)$ with the forward integration of the nonlinear dynamical model $\varphi_{t}\left(\mathscr{X}_{0}^{k}\right)$.

Compute the ensemble innovation vectors $\boldsymbol{D}^{(i)}(t)$.

Update the background perturbation matrix $A_{b}^{\prime k}$ and the term $\partial_{X} \mathbb{H} \partial_{X} \varphi_{t}\left(X_{0}\right) A_{b}^{\prime k}$.

Initialize the control variable $\delta \mathscr{Z}_{0}^{k}=\mathbf{0}$.

Inner loop: Optimization process to obtain $\widehat{\delta \mathscr{Z}_{0}^{k}}$

- Optimize the objective functional (24) in parallel

by gradient-descent method.

Do a control variable transformation

$$
\delta \mathscr{X}_{0}^{k}=\boldsymbol{A}_{b}^{\prime k} \widehat{\boldsymbol{\delta} \mathscr{Z}_{0}^{k}} .
$$

Update the initial condition and ensemble for next outer loop iteration

$$
\mathscr{X}_{0}^{k+1}=\mathscr{X}_{0}^{k}+\delta \mathscr{X}_{0}^{k} .
$$

Check the convergence condition for outer loop.

\section{end}

The analysis initial state can be propagated to the initial time and set as the background condition for the next assimilation window.

\section{End Analysis;}

$$
\boldsymbol{A}_{b}^{\prime k+1} \approx \frac{1}{\sqrt{N-1}}\left(\boldsymbol{X}_{0}^{(1), k+1}-\left\langle\boldsymbol{X}_{0}^{k+1}\right\rangle, \ldots, \boldsymbol{X}_{0}^{(N), k+1}-\left\langle\boldsymbol{X}_{0}^{k+1}\right\rangle\right) .
$$

The schematic representation of the ensemble-based variational data assimilation described above is shown in Algorithm 1.

\section{EnVar Applied to the Upper Layers Oceanic Stream Recovery}

In this paper, we are interested in estimating two-dimensional velocity fields from an image sequence, which depicts the evolution of a scalar (density fluctuation or temperature) transported by oceanic surface stream. The dynamical model and the observation model in the EnVar context dedicated to fluid motion estimation are introduced. 


\subsection{Dynamical model}

We focus on the surface quasi-geostrophic (SQG) model in this work, which is usually encountered in the modeling of upper layers of or oceanic flows at low Rossby number (see [17]). The dynamics of the SQG model stems from the quasi-geostrophy (QG) theory. It can be reduced to the evolution of buoyancy or temperature on the sea surface, denoted by a scalar field $\theta(\boldsymbol{x}, t)$, which is transported by a $2 \mathrm{D}$ spatio-temporal velocity field $\omega(\boldsymbol{x}, t)=(u, v)^{T}$. In the inviscid case, the evolution equation of the conserved scalar field can be simply expressed as:

$$
\partial_{t} \theta+\omega \cdot \nabla \theta=0
$$

where $\nabla$ denotes the gradient operator. And the velocity function $\omega$ can be deduced from $\theta$ by Riesz transforms:

$$
\theta=(-\Delta)^{\frac{1}{2}} \psi, \omega=\left(-\partial_{y}, \partial_{x}\right) \psi
$$

where $\psi$ is the stream function and $(-\Delta)^{\frac{1}{2}}$ is the fractional Laplacian operator. Such a computation can be implemented in the Fourier domain (assuming periodic boundary conditions) using Fourier transform. According to the system (1), we consider $\theta$ as the system state variable and define the operator in the data assimilation context

$$
\mathbb{M}(\theta)=\omega \cdot \nabla \theta
$$

The integration of (27) with the initial condition $\theta_{0}$ gives the state trajectory $\theta(t)=\varphi_{t}\left(\theta_{0}\right)$. Note that the motion field $\omega(t)$ can be directly computed from $\theta(t)$ based on the operation of (28) in Fourier space.

\subsection{Observation model related to images}

In order to assimilate the image data into the dynamical model, we seek to constitute an observation model directly linking the image intensity $f(\boldsymbol{x}, t)$ and the state variable $\theta(\boldsymbol{x}, t)$. Here we introduce the optical flow constraint (OFC) equation, given by

$$
\frac{\mathrm{d} f}{\mathrm{~d} t}=\frac{\partial f}{\partial t}+\nabla f \cdot \omega=0
$$

This transport equation, which states that the material derivative of luminance function is zero, relies on the assumption of a brightness conservation along a point trajectory:

$$
f(\boldsymbol{x}+\omega \mathrm{d} t, t+\mathrm{d} t)=f(\boldsymbol{x}, t) .
$$

In general, the observed images are sampled in discrete time. Assuming that we have two successive images $f(k)$ and $f(k+1)$, the integration form of OFC equation is

$$
f(k+1)=f(k)+\int_{t_{k}}^{t_{k+1}} \nabla f \cdot \omega \mathrm{d} t .
$$


Accordingly, we have the observation model linking the OFC equation and the SQG model:

$$
\left\{\begin{array}{l}
\boldsymbol{Y}=f(k+1) \\
\mathbb{H}=f(k)+\int_{t_{k}}^{t_{k+1}} \nabla f \cdot \omega \mathrm{d} t
\end{array}\right.
$$

where $\omega$ is related to the state variable $\theta$ by (28).

Remark 1. It is necessary to outline the relation between the state variable $\theta$ and the image intensity $f$. The former is a physical scalar (e.g., buoyancy or temperature) transported by the dynamical model (27-28), while the image intensity only satisfies the optical flow constraint equation (30) derived from the brightness constancy assumption. For some kinds of image data, such as the sea surface temperature (SST) images, the intensity of these images is directly correlated to the state variable. For instance, the relationship can be expressed as $f=g(\theta)+\gamma$, where $g()$ is a linear or nonlinear function and $\gamma$ denotes the image measurement noise.

\section{Experimental Results}

\subsection{Data description}

The synthetic data set is generated from a Surface Quasi-Geostrophic (SQG) model ${ }^{1}$ provided in [19], which represents an idealized oceanic domain with periodic boundary conditions. The corresponding $64 \times 64$ pixels grid is initialized with random buoyancy fluctuations. The ground-truth state vector $\theta(\boldsymbol{x}, t)$ and the corresponding velocity field $\omega(\boldsymbol{x}, t)$ are recorded at every simulation time step. The measurements, namely the images $f(k)$, are converted from the state variable matrices. As mentioned before, the relation is expressed as $f=g(\theta)+\gamma$, where $g()$ is a scaling function projecting the buoyancy data onto intensity gray value, and $\gamma$ denotes the random white noise. The true state and the measured image at the initial sample are demonstrated in Figure 1. Note that the simulation time step $d t$ and the interval of observations $\Delta t$ are different. The time step of computational fluid dynamics is chosen to satisfy the Courant-Friedrichs-Lewy (CFL) condition, while the measurements are generated per 100 steps (i.e., $\Delta t=100 d t$ ). We use a single assimilation window with 10 observations. For numerical implementation, the forward integration is achieved by a fourth-order Runge-Kutta scheme.

The initial background condition is obtained by adding a relatively strong homogeneous Gaussian noise with variance 1 . The maximum noise-signal ratio is over $10 \%$. And the random fields with the same variance are used to generate the ensemble of the background. Different numbers of ensemble members $-N=10, N=20$ and $N=40$ - are investigated.

\subsection{Results and discussions}

The errors of the background and the estimated states (called analysis states) at the initial time $t_{0}$ are illustrated in Figure 2. The absolute differences are computed with respect to the ground-truth initial state. The homogeneous Gaussian noise of the background can be observed in Figure 2(a). As we can see, the errors are significantly reduced after analysis. With the increase of the ensemble

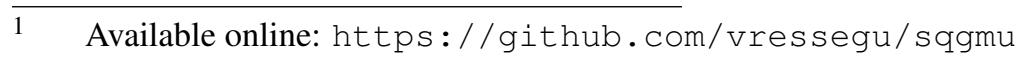




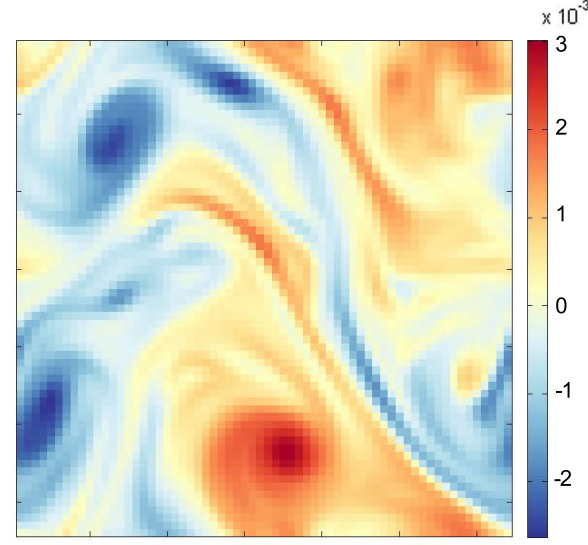

(a)

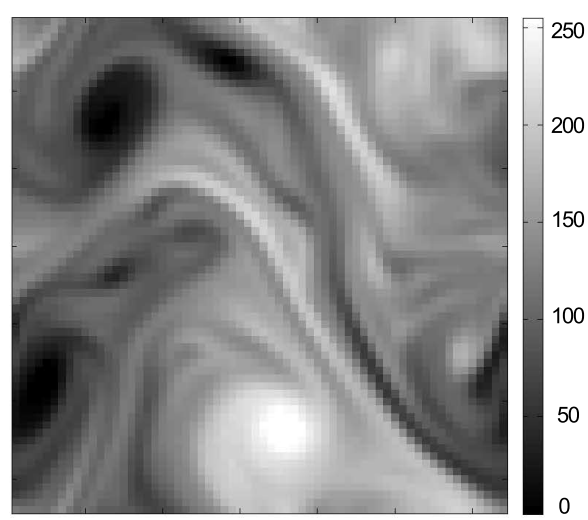

(b)

Figure 1: The true state of SQG model (a) and the measured image (b) at $t=t_{0}$.

member $N$, the accuracy of the result improves. In this case, the state matrix denoted by $\theta$ represents the buoyancy or the temperature of the flow. As indicated in the previous section, it can be converted to the velocity field by using the SQG dynamical model, which is what we are interested in. To present more details, a zoomed area with the motion fields at $t=t_{0}$ before and after the assimilation process is shown in Figure 3. Obviously, the background motion vectors provide larger angular error and magnitude error than the analysis. The estimated velocity field after data assimilation is quite consistent with the ground-truth.

We evaluate quantitatively the result with root mean square error (RMSE) of the states, which reads:

$$
R M S E=\frac{1}{n} \sqrt{\sum_{i=1}^{n}\left(\theta_{t}(\boldsymbol{x})-\theta_{e}(\boldsymbol{x})\right)^{2}},
$$

where $\theta_{t}$ and $\theta_{e}$ denote the ground-truth and the estimation (background, analysis or other estimated results), respectively. The RMSE results in the assimilation window are all plotted in Figure 4, which shows the efficiency of our EnVar method: the analysis improves the performance dramatically. We also plot the estimation error of a classical optical flow method - the Horn \& Schunck (HS) method, based on the minimization of an objective functional combining the optical flow constraint and a low velocity gradient assumption. It can be seen that the HS method is not well adapted in this fluidic case since the smoothness constraint is not consistent with the physical model. Moreover, the HS method assumes that the velocity between two successive images is constant. On the contrary, assimilationbased method can provide a continuous velocity trajectory along time since it is constrained by the dynamical flow model.

\section{Conclusions and Future Works}

In this paper, the contributions can be summarized as follows: (a) we present an ensemble-based variational data assimilation which applies a flow-dependent background error covariance and avoids the cumbersome process of constituting the tangent linear and adjoint models of standard variational data assimilation; (b) we proposed an optimal control strategy, which takes into account a fluid flow model (surface quasi-geostrophic model) and an image-based observation model (optical flow con- 


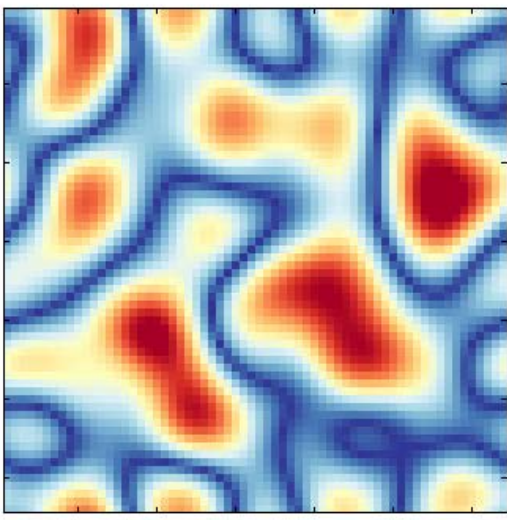

(a)

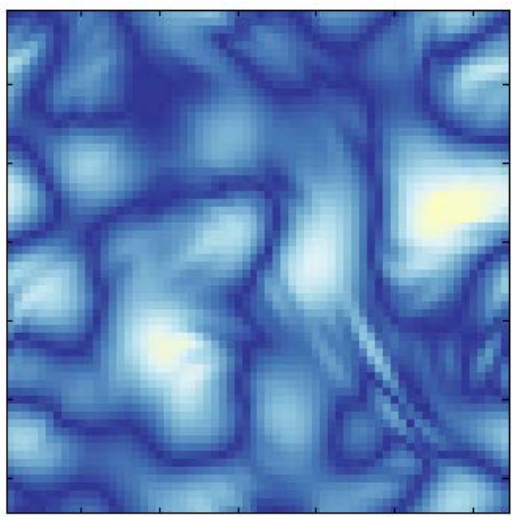

(c)

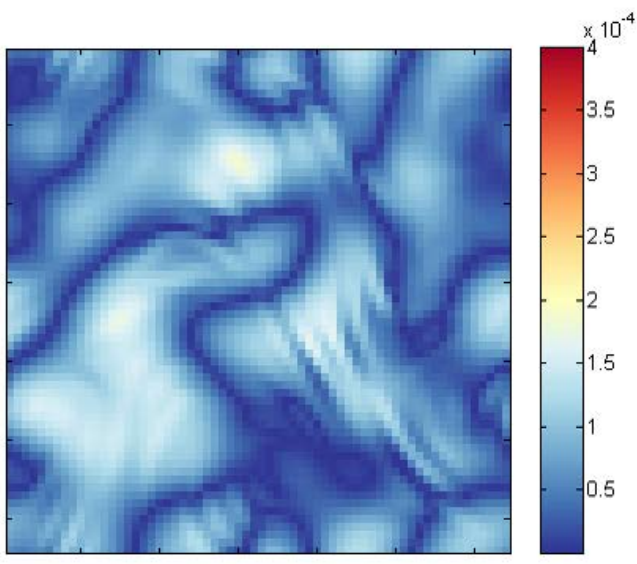

(b)

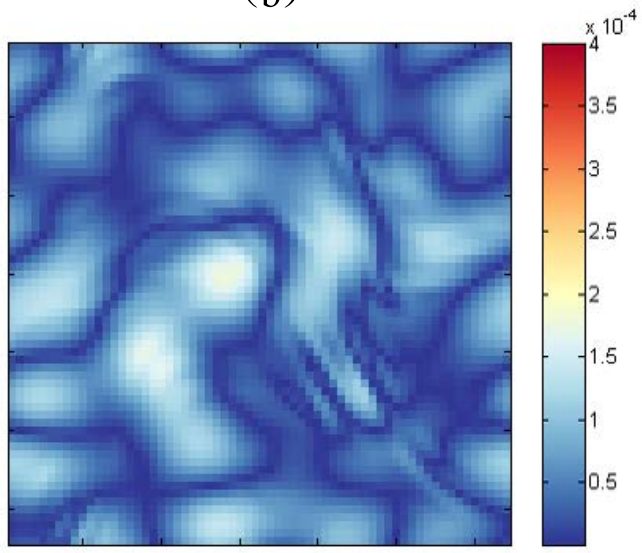

(d)

Figure 2: Error maps at $t=t_{0}$ before and after the assimilation process. The figures show the absolute differences between the true state and (a) initial background field, (b) analysis field with $N=10$, (c) analysis field with $N=20$, (d) analysis field with $N=40$.

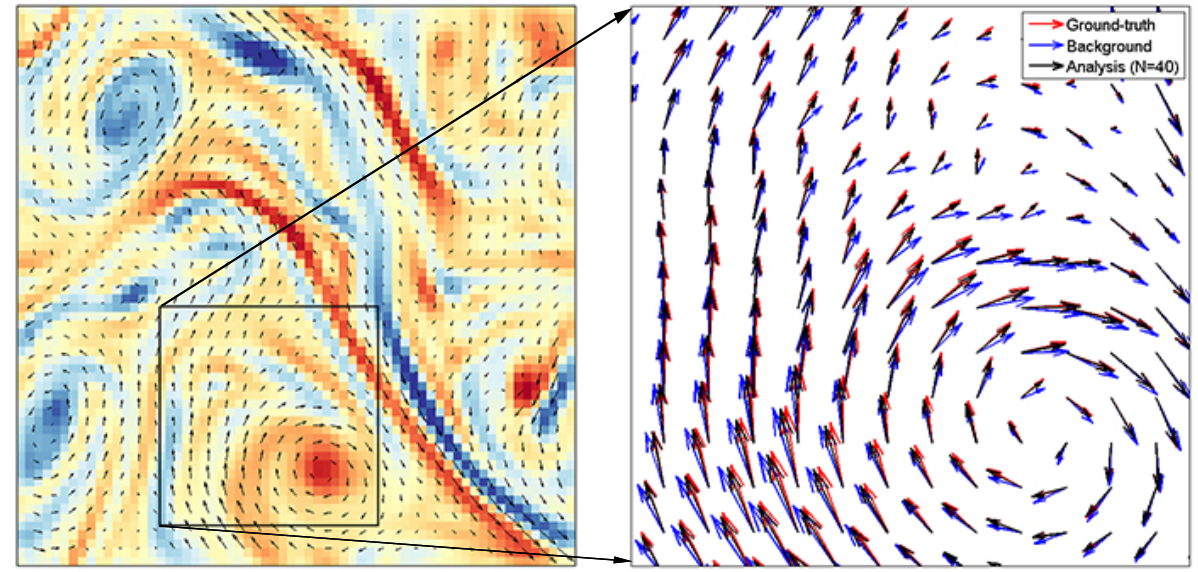

(a)

(b)

Figure 3: Velocity vectors at $t=t_{0}$ : (a) the ground-truth vorticity map; (b) the motion vectors of the ground-truth (red), the background (blue) and the analysis (black) in the zoomed area. 


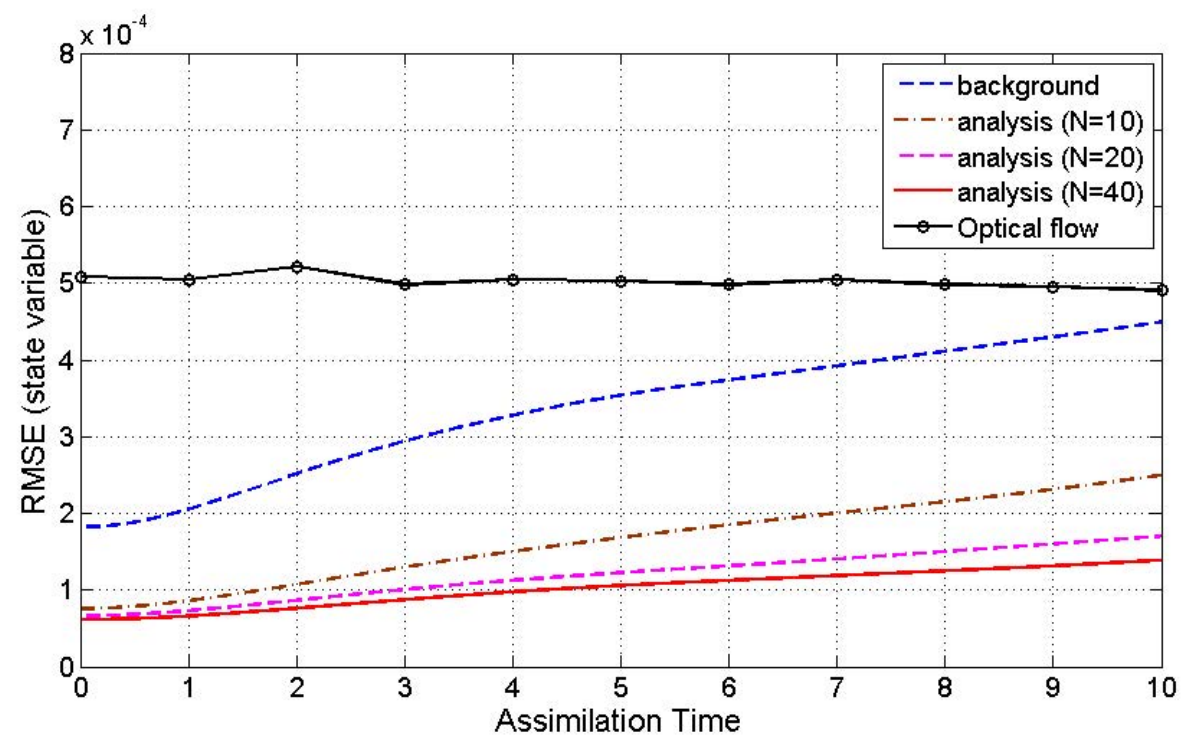

Figure 4: The RMSE comparison with respect to the ground-truth state in the assimilation window.

straint equation) in its objective functional, for fluid motion estimation problem. The experimental evaluation shows the ability of the proposed estimator to extract a dynamically consistent velocity field from noisy image observations.

There are some ongoing and future works to be done. For example, the stochastic representation of the evolution model [19] is to be investigated. By doing so, the proposed framework can be extended to more general cases for fluid motion estimation problem. Due to the stochastic model, the dynamical evolution can also be simulated on coarser grids while the resolution of the observed images is high.

\section{References}

[1] B. Horn and B. Schunck, "Determining optical flow," Artificial Intelligence, vol. 17, no. 1-3, pp. 185-203, 1981.

[2] E. Mémin and P. Pérez, "Dense estimation and object-based segmentation of the optical flow with robust techniques," IEEE Transactions on Image Processing, vol. 7, no. 5, pp. 703-719, 1998.

[3] A. Bruhn, J. Weickert, and C. Schnörr, "Lucas/Kanade meets Horn/Schunck: Combining local and global optic flow methods," International Journal of Computer Vision, vol. 61, no. 3, pp. 211-231, 2005.

[4] D. Sun, S. Roth, and M. Black, "A quantitative analysis of current practices in optical flow estimation and the principles behind them," International Journal of Computer Vision, vol. 106, no. 2, pp. 115-137, 2014.

[5] T. Corpetti, E. Mémin, and P. Pérez, "Dense estimation of fluid flows," IEEE Transactions on Pattern Analysis and Machine Intelligence, vol. 24, no. 3, pp. 365-380, 2002. 
[6] D. Heitz, E. Mémin, and C. Schnörr, "Variational fluid flow measurements from image sequences: synopsis and perspectives," Experiments in Fluids, vol. 48, no. 3, pp. 369-393, 2010.

[7] P. Héas, E. Mémin, D. Heitz, and P. Mininni, "Bayesian selection of scaling laws for motion modeling in images," in Proceedings of the IEEE 12th International Conference on Computer Vision. IEEE, 2009, pp. 971-978.

[8] X. Chen, P. Zillé, L. Shao, and T. Corpetti, "Optical flow for incompressible turbulence motion estimation," Experiments in Fluids, vol. 56, no. 1, pp. 1-14, 2015.

[9] A. Borzi, K. Ito, and K. Kunisch, "Optimal control formulation for determining optical flow," SIAM Journal on Scientific Computing, vol. 24, no. 3, pp. 818-847, 2003.

[10] N. Papadakis and E. Mémin, "Variational assimilation of fluid motion from image sequence," SIAM Journal on Imaging Sciences, vol. 1, no. 4, pp. 343-363, 2008.

[11] V. Barbu and G. Marinoschi, "An optimal control approach to the optical flow problem," Systems \& Control Letters, vol. 87, pp. 1-9, 2016.

[12] A. Cuzol and E. Mémin, "A stochastic filtering technique for fluid flow velocity fields tracking," IEEE Transactions on Pattern Analysis and Machine Intelligence, vol. 31, no. 7, pp. 1278-1293, 2009.

[13] F.-X. Le Dimet and O. Talagrand, "Variational algorithms for analysis and assimilation of meteorological observations: theoretical aspects," Tellus A: Dynamic Meteorology and Oceanography, vol. 38, no. 2, pp. 97-110, 1986.

[14] C. Liu, Q. Xiao, and B. Wang, "An ensemble-based four-dimensional variational data assimilation scheme. Part I: Technical formulation and preliminary test," Monthly Weather Review, vol. 136, no. 9, pp. 3363-3373, 2008.

[15] Y. Yang, C. Robinson, D. Heitz, and E. Mémin, "Enhanced ensemble-based 4DVar scheme for data assimilation," Computers \& Fluids, vol. 115, pp. 201-210, 2015.

[16] I. M. Held, R. T. Pierrehumbert, S. T. Garner, and K. L. Swanson, "Surface quasi-geostrophic dynamics," Journal of Fluid Mechanics, vol. 282, pp. 1-20, 1995.

[17] G. Lapeyre and P. Klein, "Dynamics of the upper oceanic layers in terms of surface quasigeostrophy theory," Journal of Physical Oceanography, vol. 36, no. 2, pp. 165-176, 2006.

[18] G. Burgers, P. Jan van Leeuwen, and G. Evensen, "Analysis scheme in the ensemble Kalman filter," Monthly weather review, vol. 126, no. 6, pp. 1719-1724, 1998.

[19] V. Resseguier, E. Mémin, and B. Chapron, "Geophysical flows under location uncertainty, Part II: Quasigeostrophic models and efficient ensemble spreading," Geophysical \& Astrophysical Fluid Dynamics, 2017. 